\title{
The generalized criterion of multiaxial random fatigue based on conception proposed by prof. Macha
}

\author{
Tadeusz Łagoda ${ }^{1}$, Marta Kurek $^{1}$, and Karolina Lagoda ${ }^{2}$ \\ ${ }^{1}$ Department of Mechanics and Machine Design, Opole University of Technology, 5 Mikołajczyka \\ Street, 45-271 Opole, Poland \\ ${ }^{2}$ Department of Mechanics, Materials Science and Engineering, Wrocław University of Science and \\ Technology, Smoluchowskiego Street 25, Wrocław, Poland
}

\begin{abstract}
This criterion has been repeatedly verified, analyzed and special cases of this criterion reducing complex stress to equivalent uniaxial were taken into account. Since both normal and shear stress are vectors, we encounter the mathematical problem of adding these vectors, and the question arises how to understand the obtained equivalent stress, because two perpendicular vectors are added with weighting factors. Therefore, in this work it was proposed to adopt a system of complex numbers. Normal stress was defined as the real part and shear stress as imaginary part. As a result, on the basis of the defined complex number and basing on pure bending and pure torsion after transformations, the expression for equivalent stress was identical to the previously proposed criteria defined on the basis of the concept of prof. Macha.
\end{abstract}

\section{Introduction}

The criteria for multi-axial fatigue in complex stress states have been proposed for many years and so far no universal one has been found for use in practice. Typically, stress, strain and energy (stress-strain) criteria are applied. The most commonly used criteria in both statics and fatigue are stress criteria. They are also preferred by engineers when estimating the fatigue life of structural components. According to these criteria, formulas for the equivalent value are derived. The most commonly used stress criteria are those recorded in the critical plane, which are based on normal and shear stress. These values are vectors perpendicular to each other, so adding them as a scalar is a significant problem of mathematical correctness. Numerous authors successfully apply these criteria, but no one wonders about the physical meaning of adding such vectors.

The aim of the present paper is to present the expression on the value of equivalent stress, which is consistent with the principles of mathematics. In our deliberations it was assumed that the stress in the plane is a complex number defined in such a way that the normal stress is the real part of this number, and the shear stress is the imaginary part. 


\section{Multiaxial fatigue criteria in critical plane}

In the event of multi-axis random loads, the calculation of fatigue life consists in the reduction of triple-axis or double-axis tension state to the equivalent single-axis state by means of proper fatigue criteria. The critical plain concept assumes that material fatigue crack is caused by tensions in the material (critical) plain. A source of this assumption is the observation of cracks in metals which occur in specific plains. The application of critical plain in the description of multi-axis fatigue was proposed, for the first time, by Stanfield in 1935 [1]. His theory of the combination of normal and shear stress in a set (critical) plain with a specific orientation was not proved experimentally. An expression for the critical value of shear stress:

$$
\tau_{c}=\tau_{n s, a}+k \sigma_{n, a}
$$

where $k$ is a material constant, was not verified.

Next, Stulen and Cummings [2] used the expression "critical plain" with reference to the criterion of multi-axis material fatigue devised in 1954. The criterion was based on the following two assumptions:

1. The fatigue strength of polycrystallic metal depends on the critical tangential tension which is the linear function of the maximum normal tension in the critical plain;

2. Fatigue strength does not depend on the average value of shear stress in the critical plain.

Critical shear stress $\tau_{c}$ is calculated from the following dependence

$$
\tau_{c}=\tau_{c 0}+b \sigma_{n}
$$

where: $\tau_{c 0}$ is a material constant determined for $\sigma_{n}=0 ; b$ is a coefficient determining metal sensitivity to the impact of normal tension $(b<0)$. As far as ductile metals are concerned, $b$ coefficient is close to zero. Whereas, as far as brittle metals are concerned, b coefficient reaches considerably high negative values. Stulen and Cummings did not precise the expressions 'ductile metals' and 'brittle metals'. Critical plain orientation is determined by the maximum quotient of shear stress $\tau_{\eta \text { s }}$ and critical shear stress $\tau_{c}$

$$
\frac{\tau_{n s}}{\tau_{c}}=\frac{\tau_{n s}}{\tau_{c 0}+b \sigma_{n}}
$$

Material destruction will occur if shear stress $\tau_{\eta s}$ in critical plain reaches critical value $\tau_{c}$

$$
\tau_{n s}=\tau_{c}
$$

It is only in Findley's work from the 1950s that we can find further mentions about the critical plane. Author [3] argued that the main cause of material fatigue is variable shear stress with the participation of normal stresses in the critical plane. Findley adapted Stulen's and Cummings' proposal for a combination of cyclic proportional loading: tension-compression (bending), and torsion taking into account the value average load different to 0. Eventually, Findley developed Stanfield's concepts and proposed a criterion in which he took into account the maximum value of normal stress. 


$$
\tau_{c}=\tau_{n s, a}+k \sigma_{n, \max }
$$

According to Findley, the location of the critical plane at zero values of the average stresses depends on the location of the maximum stress direction $\sigma_{l}$ and the material constant $k$. Constant $k$ is a function of the number of cycles to destruction and the type of material. According to Findley, the critical plane is the plane on which the linear combination of stresses reaches the maximum.

In his works [4, 5], McDiarmid investigated the influence of normal and shear stresses acting in a plane perpendicular to the plane with maximum shear stress for fatigue durability. Further analysis [6] resulted in a new expression for equivalent stress depending on the method of material cracking. The criterion was based on the assumption that fatigue damage is caused by a linear combination of normal and shear stresses in the critical plane defined by the maximum value of shear stress

$$
\tau_{\text {aeq }}=k_{1} \sigma_{n, \max }+\tau_{\max },
$$

where:

$\sigma_{n, \max }$ - maximum normal stress in the plane of maximum shear stress,

$\tau_{\max }$ - shear stress in the plane of maximum shear stress,

$k_{1}$ - material constant defining part of normal stresses.

Critical plane is the plane with the maximum value of the tangential stress amplitude.

Matake [7] presented a criterion similar to Findley's expression assuming that the fatigue stress of the material corresponds to the maximum shear stress involving normal stress in the plane of maximum shear stress amplitude in accordance with

$$
\tau_{n, s}+k \sigma_{n, \max } \leq \tau_{a f}
$$

This criterion was developed for analyzing torsion, bending and the combination of proportional torsion and bending. The factor $k$ can be determined from the limits of fatigue according to the formula::

$$
k=2 \frac{\tau_{a f}}{\sigma_{a f}}-1 .
$$

The critical plane in this criterion is the plane of maximum shear stress.

Papadopoulos $[8,9]$ proposed a criterion which is a linear combination of the maximum amplitude of generalized shear stress $T_{\sigma}$ in the critical plane and the maximum value of hydrostatic stress $\sigma_{H}$ in the mesoscopic scale, which can be written as

$$
\max T_{\sigma}+k \sigma_{H, \max } \leq \tau_{a f},
$$

where $k$ is a coefficient determined from uniaxial fatigue tests in the form:

$$
k=3 \frac{\tau_{a f}}{\sigma_{a f}}-\frac{1}{2}
$$

This approach is a summary hypothesis used in the group of criteria based on invariants of the stress state with that used in criteria based on the values of average stresses and the approach related to the concept of the critical plane. The amplitude of shear stress is 
defined as the average value of stresses in all directions. The maximum value of this stress determines the orientation of the critical plane.

In 1996 Vidal, Kenmeugne, Robert i Bahuaud [10] presented a criterion based on the critical plane, taking into account the amplitude of the equivalent stress course $\sigma_{e q, a}$, shear stress amplitude $\tau_{\eta s, a}$, normal stress amplitude $\sigma_{\eta a}$ and average normal stress amplitude $\sigma_{\eta, m}$ in the critical plane. The amplitude and avarage value of normal stress are added taking into account the coefficients $a(N)$ and $b(N)$ determined on the basis of uniaxial fatigue tests and dependent on the number of cycles to destruction of $N$

$$
\sigma_{e q, a}=\tau_{\eta s, a}+a(N) \sigma_{\eta, a}+b(N) \sigma_{\eta, m}
$$

The location of the critical plane is determined by the maximum value of the amplitude of the equivalent stress $\sigma_{e q}$. The validity of the presented model has been proved on the basis of tests in the range of consistent and incompatible in the phase cyclic loads of different geometries of the samples.

As shown in formulas (1) - (11), authors often use the addition of amplitudes of shear and normal stresses in a given critical plane differently defined $[11,12]$.

\section{The criterion of multi-axial fatigue according to the concept of prof. Macha}

Macha [13] formulated the criterion of maximum normal and tangential tensions in scrap plain which is the generalisation of the scope of the random loads of many criteria.

The assumptions of this criterion are as follows:

1. Fatigue scrap is formed under the influence of normal stress $\sigma_{\eta}(t)$ and shear stress $\tau_{\eta s}(t)$ in the $S$ direction on the scrap plain with normal $\eta$ in the following combination

$$
\tau_{\eta s}(t)+K \sigma_{\eta}^{r}(t)
$$

2. The $S$ direction in the scrap plain coincides with the average direction of maximum shear stress $\tau_{\eta \operatorname{smax}}(t)$.

3. In the limit state for given fatigue life, the maximum value of the linear combination of tensions $\tau_{\eta s}(t)$ and $\sigma_{\eta}(t)$ and in the conditions of multi-axis random loads is expressed with the following formula

$$
\max _{t}\left\{\tau_{\mathrm{\eta} s}(t)+K \sigma_{\mathrm{\eta}}^{r}(t)\right\}=F,
$$

where: $K, r, F$ - material constants determined in the conditions of sine loads.

Later on, the expression (13) was analysed spectrally and linearly, in the form of

$$
\max _{\mathrm{t}}\left\{B \tau_{\eta \mathrm{s}}(t)+K \sigma_{\eta}(t)\right\}=F
$$

where: $B, K, F$-constants used for the selection of a specific criterion form.

The equivalent value of stress according to criterion (14) may be recorded in amplitudes as 


$$
\sigma_{\mathrm{aeq}}=B \tau_{\mathrm{a \eta s}}+K \sigma_{\mathrm{a \eta}}
$$

It is a linear type due to the tension state constituents. The plain positioning of fatigue scrap is described by means of the average values of main tension directional cosines. The following three methods are suggested for determining the expected critical plain direction: weight functions method, variance method and damage accumulation method.

Ogonowski [14] defined the form of the stress criterion in the plane of maximum normal stresses as a particular form of the Macha expression (Eq. 14) written as

$$
\sigma_{e q}(t)=B \tau_{\eta \mathrm{n}}(t)+\max _{t}\left\{\sigma_{n}(t)\right\}
$$

This hypothesis is intended for brittle materials, and the critical plane was determined by the maximum value of normal stresses. Parameter $B$ is selected on the basis of the best compatibility of calculation results for a given material in the case of loads non-compliant in the phase. The second proposition of Ogonowski's criterion determined the critical plane by the maximum value of shear stresses and was written as

$$
B \max _{t}\left\{\tau_{\mathrm{\eta} s}(t)\right\}+K \sigma_{\mathrm{\eta}}(t)=f\left(N_{f}\right)
$$

The discussed criteria in the strain and energy notation were described and verified in paper [15].

Walat [16] proposed a criterion for materials characterized by intermediate properties between elastic-brittle and elastic-plastic materials

$$
\sigma_{e g}(t)=\frac{4 \sqrt{3}+3 \sqrt{2} k}{3(\sqrt{3} \pm 1)} \sigma_{\eta}(t)+\frac{\sqrt{3}(3 \sqrt{2} k-4)}{3(\sqrt{3} \pm 1)} \tau_{\eta s}(t)
$$

where we are dealing with such a definition of the critical plane in which elements of the covariance matrix of normal and shear stresses occur, i.e. the plane in which the maximum covariance of shear and normal stresses occurs.

In the plane of the maximum value of maximum shear stress (17) the value for the equivalent expression takes the form [14]

$$
\sigma_{e q}(t)=\left(2-\frac{\sigma_{a f}}{\tau_{a f}}\right) \sigma_{n}(t)+\frac{\sigma_{a f}}{\tau_{a f}} \tau_{n s}(t)
$$




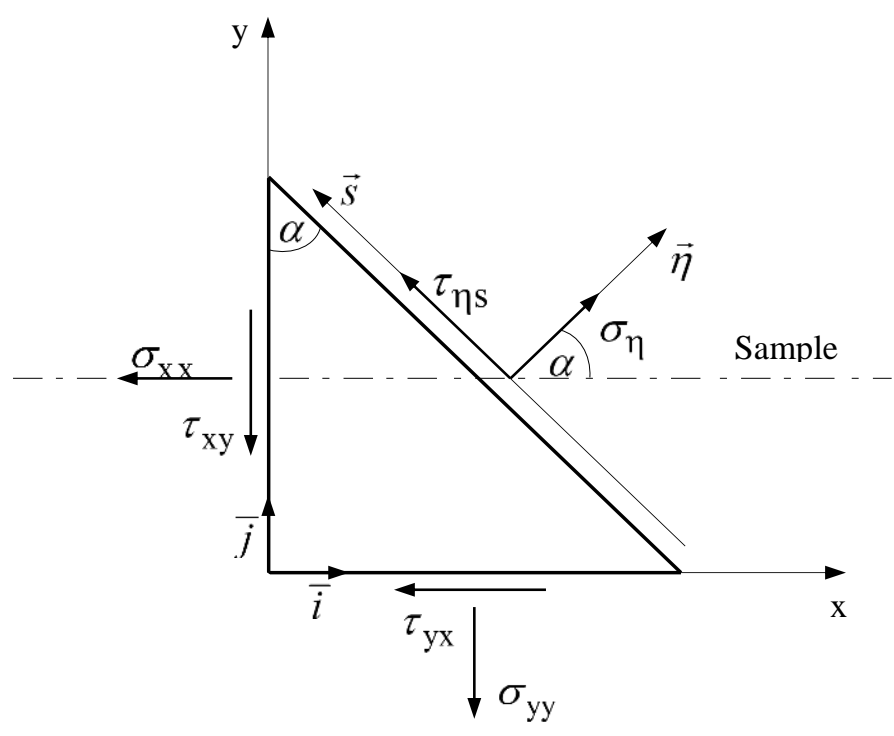

Fig. 1. The interpretation of the critical plain positioning

Here, it should be noted that in the proposed notation there are two stresses: normal $\sigma_{\mathrm{xx}}(\mathrm{t})$ and shear $\tau_{x y}(t)$, as shown in Fig.1. In the critical plane at an angle $\beta$ these stresses are directed at each other at right angles. Therefore, there is a mathematical doubt about the addition of vectors perpendicular to each other, because it should be remembered that these stresses are of a vector character. So the question arises what physical quantity we get in effect. It is known from experience that this proposition is successfully used according to this criterion and similar other criteria on in the critical plane where both normal and shear stress are taken into account [17-24].

\section{Criterion of multi-axial fatigue in the critical plane in the notation of complex numbers}

The expression on the course of the equivalent stress according to the proposed criterion of multi-axis random fatigue in the inscription in complex numbers can be written in the general formula as

$$
\sigma_{e q}(t)=|p(t)|(A \cos \varphi+B i \sin \varphi) .
$$

The graphic interpretation of the expression (20) is shown in Figure 2. 


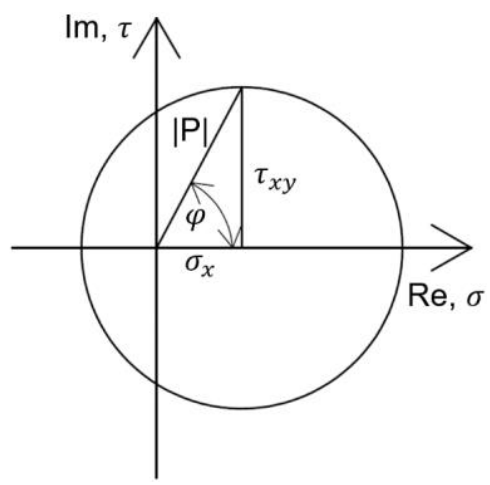

Fig. 2. Interpretation of stress state components in the critical plane as a complex number

The proposed model uses equivalent stress as a complex number, where the value of normal stress is the real part, and the value of shear stress is the imaginary part. The above dependence must be true for simple stress states, i.e. tension-compression (pendulum bending) and shearing (double-sided torsion). Therefore, we get two equations and thus one can determine two material constants $A$ and $B$, which occur in equation (20).

In the case of pure tension-compression (pendulum bending) as shown in Fig. 3a, based on the formula (20), we get

$$
\sigma_{e q}(t)=\left|\sigma_{x}(t)\right|(A+B i)
$$

and pure shear (torsion) on the basis Fig.3b and formula (20) we obtain

$$
\sigma_{e q}(t)=\left|\tau_{x y}(t)\right|(B i)
$$

After the transformations of the system of equations (21) and (22) and changing to amplitudes, assuming in the extreme situation the limit of fatigue for tension-compression $\sigma_{\mathrm{af}}$ and shear $\tau_{\mathrm{af}}$, we obtain the same formula to the formula (19).

a)

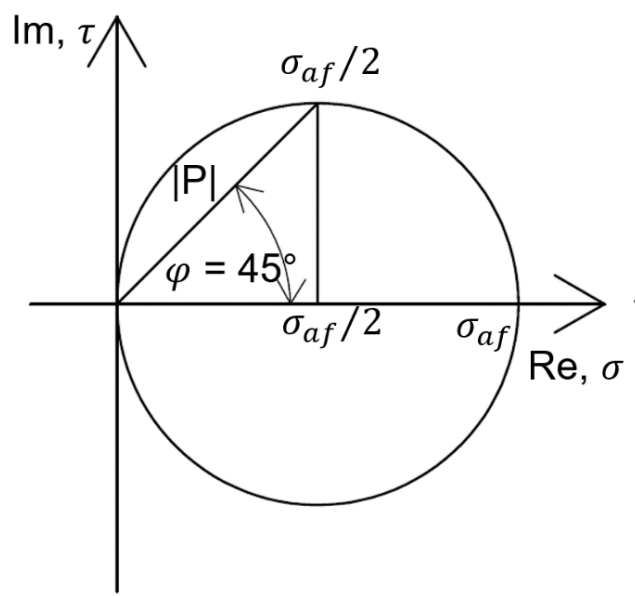

b)

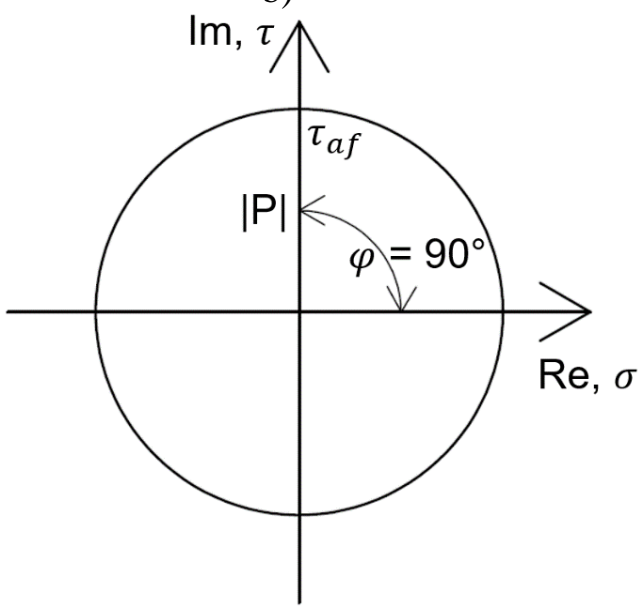

Fig. 3. The stress in the critical plane as a complex number for a) stretching, b) shearing 


\section{Summary and conclusions}

From a brief review of the literature, it can be noted that very often we are dealing with the addition of mutually perpendicular vectors of normal and shear stress. Although this is inconsistent with the principles of mathematics, this approach gives good results in estimating fatigue life. It is no different in the case of the criterion based on the concept of prof. Macha. It turned out that treating stress in the critical plane as a complex number on the assumption that normal stress is the real part and shear stress is the imaginary part resulting in an expression identical to Macha's proposal, which is constantly developed mainly at the Opole University of Technology in the Department Mechanics and Machine Design.

Acknowledgement: The authors gratefully acknowledge the support by the Polish National Science Centre (No. 2016/21/D/ST8/02007)

\section{References}

1. G. Stanfield, Discussion of „The strength of metals under combined alternating stresses", by H.Gough and H.Pollard. Proc. Inst. of Mechanical Engineers, 131, 93 (1935)

2. F. Stulen, H. Cummings, A failure criterion for multi-axial fatigue stresses. Proceedings-American Society for Testing and Materials, 19428-2959, p. 822-835 (1954)

3. W.N. Findley, A theory for the effect of mean stress on fatigue of metals under combined torsion and axial load or bending. Journal of Engineering for Industry, pp. 301-306 (1959)

4. D. Mcdiarmid, A general criterion for high cycle multiaxial fatigue failure. Fatigue \& Fracture of Engineering Materials \& Structures, 14, p. 429-453 (1991)

5. D. Mcdiarmid, A new analysis of fatigue under combined bending and twisting, The Aeronautical Journal, 78, p. 325-329 (1974)

6. D. Mcdiarmid, Multiaxial fatigue life prediction using a shear stress based critical plane failure criterion, Technical Research Centre of Finland, Fatigue Design,2, (1992)

7. T. Matake, An explanation on fatigue limit under combined stress. Bulletin of JSME, 20, p. 257-263 (1977)

8. I.V. Papadopoulos, Long life fatigue under multiaxial loading. International Journal of Fatigue, 23, p. 839-849(2001)

9. I.V. Papadopoulos, P. Davoli, C. Gorla, M. Filippini, A. Bernasconi, A comparative study of multiaxial high-cycle fatigue criteria for metals. International Journal of Fatigue, 19, p.219-235(1997)

10. E. Vidal, B. Kenmeugne, J. Robert, J. Bahuaud, Fatigue life prediction of components by the use of multiaxial criterion. In: ICBMFF4, (1994)

11. G. Marulo, F Frendo, L. Bertini, A. Fatemi, On the application of a critical plane approach to the life assessment of welded joints. Procedia Engineering, 213, p. 448458(2018) 
12. S. Vantadori, G.M.J. Almedia, G. Fortese, G.C.V Pessoa J.A Araujo, Early fretting crack orientation by using the critical plane approach. International Journal of Fatigue, 114 p. 282-288(2018)

13. E. Macha. Generalization of fatigue fracture criteria for multiaxial sinusoidal loadings in the range of random loadings, Biaxial and Multiaxial Fatigue, EGF3 (Edited by. M.W.Brown and K.J.Miller), Mechanical Engineering Publications, London, p.425436 (1989)

14. T. Lagoda, P. Ogonowski, Criteria of multiaxial random fatigue based on stress, strain and energy parameters of damage in the critical plane, Mat.-wiss. u. Werkstofftech, 36, p.429-437 (2005)

15. K. Walat M. Kurek, P. Ogonowski, T. Łagoda, The multiaxial random fatigue criteria based on strain and energy damage parameters on the critical plane for the low-cycle range. International Journal of Fatigue, 37 p.100-111 (2012)

16. K. Walat, T. Łagoda, The equivalent stress on the critical plane determined by the maximum covariance of normal and shear stresses. Materialwissenschaft und Werkstofftechnik, 41, p. 218-220 (2010)

17. A. Niesłony, T. Łagoda, K. Walat, M. Kurek, Multiaxial fatigue behaviour of AA6068 and AA2017A aluminium alloys under in-phase bending with torsion loading condition, Mat.-wiss. U. Werkstofftech., 45, p.947-952 (2015)

18. K. Walat, T. Łagoda, M. Kurek, Life time assessment of an aluminium alloy under complex low cycle fatigue loading, Materials Testing, 57, p.160-164 (2015)

19. M. Kurek, T. Łagoda, S. Vantadori, Estimation of fatigue life of selected construction materials under cyclic loading, Fracture and Structural Integrity, IX, p.302-308 (2015)

20. A. Karolczuk, M. Kurek, T. Lagoda, Fatigue life of aluminium alloy 6082 T6 under constant and variable amplitudę bending with torsion, J. of Theoretical and Applied Mechanics, vol.53, No 2, 2015, pp.421-430

21. C. Ronchei, A. Carpinteri, G. Fortese, A. Spagnoli, S. Vantadori, M. Kurek, T. Łagoda, Life estimamtion by varying the critical plane orientation in the modified CaripnteriSpagnoli criterion, Frattura ed Integrita Strutturale, 34, p.74-79 (2015)

22. M. Kurek, T. Łagoda, F. Morel, Estimation of the fatigue life of $35 \mathrm{NCD} 16$ alloy steel under random loading, Materials Science, 52, p.492-499 (2017)

23. A. Carpinteri, M. Kurek, T. Łagoda, S. Vantadori, Estimation of fatigue life under multiaxial loading by varying the critical plane orientation, Int. J. Fatigue, 100, part II, p.512-520 (2017)

24. M. Kurek, T. Łagoda, Determination of the critical plane orientation depending on the fatigue curves for bending and torsion, Frattura ed Integrita Strutturale, 41, p.24-30 (2017) 